\title{
Structural and functional brain-wide alterations in A350V lqsec2 mutant mice displaying autistic-like behavior
}

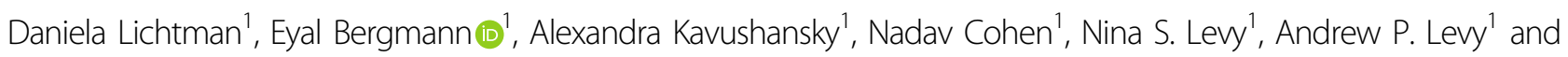
Itamar Kahn (1)

\begin{abstract}
IQSEC2 is an X-linked gene that is associated with autism spectrum disorder (ASD), intellectual disability, and epilepsy. IQSEC2 is a postsynaptic density protein, localized on excitatory synapses as part of the NMDA receptor complex and is suggested to play a role in AMPA receptor trafficking and mediation of long-term depression. Here, we present brainwide structural volumetric and functional connectivity characterization in a novel mouse model with a missense mutation in the IQ domain of IQSEC2 (A350V). Using high-resolution structural and functional MRI, we show that animals with the A350V mutation display increased whole-brain volume which was further found to be specific to the cerebral cortex and hippocampus. Moreover, using a data-driven approach we identify putative alterations in structure-function relations of the frontal, auditory, and visual networks in A350V mice. Examination of these alterations revealed an increase in functional connectivity between the anterior cingulate cortex and the dorsomedial striatum. We also show that corticostriatal functional connectivity is correlated with individual variability in social behavior only in A350V mice, as assessed using the three-chamber social preference test. Our results at the systemslevel bridge the impact of previously reported changes in AMPA receptor trafficking to network-level disruption and impaired social behavior. Further, the A350V mouse model recapitulates similarly reported brain-wide changes in other ASD mouse models, with substantially different cellular-level pathologies that nonetheless result in similar brain-wide alterations, suggesting that novel therapeutic approaches in ASD that result in systems-level rescue will be relevant to IQSEC2 mutations.
\end{abstract}

\section{Introduction}

Autism spectrum disorder (ASD) is a neurodevelopmental disorder with a highly genetically heterogeneous component ${ }^{1,2}$. It is diagnosed on the basis of a combination of behavioral observations and clinical interviews that assess deficits in social interactions, communication and language, as well as repetitive and stereotyped behaviors ${ }^{3}$, and is found to be associated with other cognitive and neurological conditions ${ }^{4,5}$. Despite the complexity of ASD, genetic research has contributed significantly to the

Correspondence: Andrew P. Levy (alevy@technion.ac.il) or

Itamar Kahn (kahn@technion.ac.il)

${ }^{1}$ Rappaport Faculty of Medicine, Technion-Israel Institute of Technology, Haifa 31096, Israel elucidation of its pathophysiology ${ }^{1}$. Research in genetically heterogeneous ASD populations has demonstrated converging pathophysiology, pointing out the role of glutamatergic cortical synapses in the pathology ${ }^{6,7}$. At the systems level, findings in humans and genetic animal models have implicated disrupted brain-wide connectivity in ASD pathophysiology with diametrically different effects on distal versus local connectivity ${ }^{8,9}$.

Intrinsic functional connectivity MRI (fcMRI), the temporal correlation of spontaneous blood oxygenation level-dependent (BOLD) signal fluctuations in the brain, has been shown to be a useful method for characterization of brain networks in humans and animals ${ }^{10-14}$. Several studies have demonstrated that fcMRI can be used to

\section{(c) The Author(s) 2021}

(c) (i) Open Access This article is licensed under a Creative Commons Attribution 4.0 International License, which permits use, sharing, adaptation, distribution and reproduction cc) in any medium or format, as long as you give appropriate credit to the original author(s) and the source, provide a link to the Creative Commons license, and indicate if changes were made. The images or other third party material in this article are included in the article's Creative Commons license, unless indicated otherwise in a credit line to the material. If material is not included in the article's Creative Commons license and your intended use is not permitted by statutory regulation or exceeds the permitted use, you will need to obtain permission directly from the copyright holder. To view a copy of this license, visit http://creativecommons.org/licenses/by/4.0/. 
identify functional alterations in human diseases and in transgenic models in rodents ${ }^{9,15-17}$. Specifically, fcMRI studies in several ASD animal models have detected functional connectivity alterations that are consistent with electrophysiological recordings and human fcMRI stu$\operatorname{dies}^{17-23}$.

IQSEC2, an X-linked gene coding for a protein found in the postsynaptic density of glutamatergic synapses ${ }^{24}$, has been implicated in the trafficking of AMPA receptors and regulation of synaptic transmission ${ }^{25-28}$. In humans, mutations in the IQSEC2 gene are associated with ASD, intellectual disabilities, and epilepsy ${ }^{29-32}$. We recently described a novel mouse model ${ }^{28}$ with a missense mutation in the IQ domain of Iqsec 2 at amino acid residue 350 resulting in a valine for alanine substitution (A350V). The A350V mouse model was generated using CRISPR technology based on a human de novo mutation $^{32}$ and demonstrated a significant reduction in the surface expression of GluA2 AMPA receptors in hippocampal neurons along with increased locomotion activity and abnormal social behavior. Further, a more recent study that thoroughly characterized social behaviors in this model found that A350V mice display deficits in sex preference and emotional state preference behaviors ${ }^{33}$. Collectively, these findings demonstrate that the A350V mouse model manifests some of the abnormalities found in the human condition that inspired the generation of this mouse model.

Here, we investigated the impact of the A350V mutation on brain-wide structural and functional alterations using MRI. We examined the A350V mouse model using a structure-function analysis that allowed us to characterize putative changes in functional connectivity in a hypothesis-free data-driven approach. Further, we tested whether the observed functional connectivity alterations can explain behavioral variability, thereby linking functional connectivity changes to ASD-related behavioral impairments. By characterizing brain-wide changes in functional connectivity and further investigating the altered regions, we found that the A350V mouse model presents increased corticostriatal functional connectivity which is linked to abnormal social behavior in the threechamber sociability task.

\section{Materials and methods \\ Ethics}

All animal experiments were conducted in accordance with the United States Public Health Service's Policy on Humane Care and Use of Laboratory Animals and approved by the Institutional Animal Care and Use Committee of the Technion-Israel Institute of Technology.

\section{Animals and housing conditions}

A350V Iqsec 2 mice on a C57Bl/6J background were generated by CRISPR as previously described ${ }^{28}$. A350V Iqsec2 hemizygous male mice and male wild-type (WT) littermates were housed in groups of 2-5 animals per cage in a reversed 12-h light-dark cycle with food and water available ad libitum. The housing room was maintained at $23 \pm 2{ }^{\circ} \mathrm{C}$. All experiments were conducted during the dark phase.

\section{Experimental design}

The experimental protocol is presented in Fig. 1. Behavioral experiments were performed on 6-7-week-old mice in which all animals were tested for social preference $\left(n_{\mathrm{WT}}=18, n_{\mathrm{A} 350 \mathrm{~V}}=14\right)$. Weights were equivalent for the A350V (21.342 $\pm 1.158 \mathrm{~g}[$ mean \pm SD] $)$ and WT $(21.838 \pm$ $1.945 \mathrm{~g}$ ) cohorts. A subset of the cohort then underwent a head-post surgery at 10-12 weeks of age and was allowed

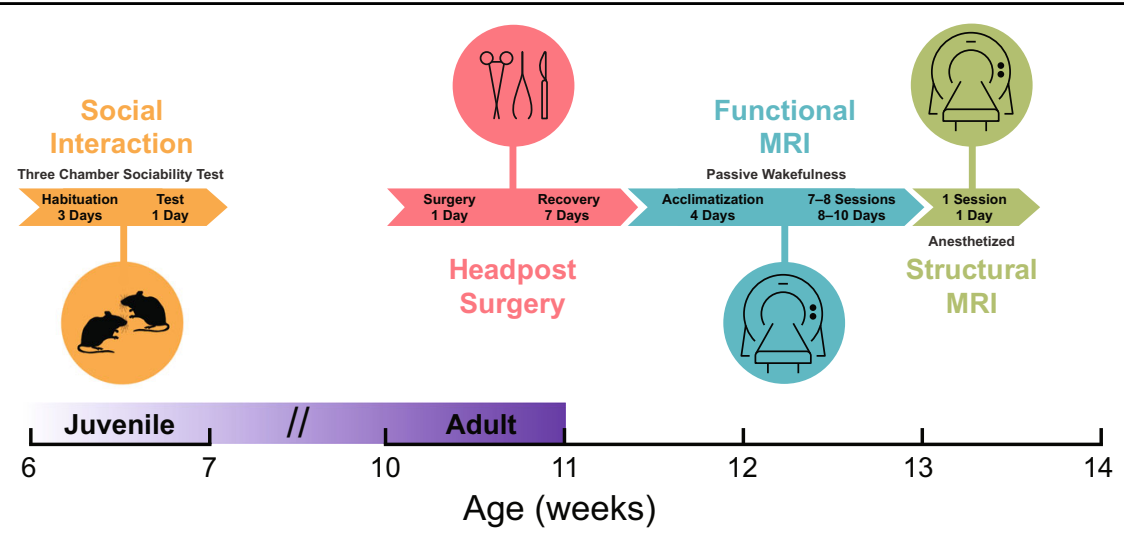

Fig. 1 Experimental protocol. The timeline shows the sequence of experimental steps animals underwent. Social interaction assessment was examined on juvenile mice. At adulthood, the mice underwent a head-post surgery, were allowed to recover, and underwent a 4 days acclimatization period to awake head-fixed imaging followed by multiple functional MRI sessions for each animal. Finally, a single structural MRI was acquired. 
to recover for 1 week in their home cage. Two mice (one of each genotype) died during the surgery and, therefore, two additional mice, who did not participate in the behavioral experiments, were operated on to replace these mice $\left(n_{\mathrm{WT}}=13, n_{\mathrm{A} 350 \mathrm{~V}}=13\right)$. To reduce stress and head movement during fcMRI scanning, mice were acclimatized to the head fixation position inside the scanner for 4 days prior to the first fcMRI $\operatorname{scan}^{34}$. Thus, at 3-4 months of age, the mice underwent 7-8 awake headfixed fcMRI sessions (one session per day) which were followed with a single high-resolution structural imaging scan under anesthesia on the last fcMRI session. Weights at the timepoint of the first fcMRI session were equivalent for A350V $(27.846 \pm 1.549 \mathrm{~g})$ and WT $(27.592 \pm 1.546 \mathrm{~g})$.

\section{Three-chamber sociability test}

Social interaction was measured using the three-chamber test $^{35}$ in order to assess autistic-like behavior. Subject mice were assessed for the tendency to prefer an unfamiliar conspecific mouse (social stimulus; Stranger 1) over a novel object. Mice (subject and stimulus) were habituated to the testing room for three consecutive days prior to the test day for $1 \mathrm{~h}$. Stimulus mice were further habituated to the wire cages $(10.8 \mathrm{~cm}$ in height and $10.2 \mathrm{~cm}$ diameter at the bottom; Galaxy Cup, Spectrum Diversified Designs, Inc., Streetsboro, OH, USA) for 20 min each day. On the test day, subject mice were habituated to the apparatus $(70 \times 29 \times 35$ $\mathrm{cm}^{3}$ ) for $10 \mathrm{~min}$ and were able to explore all three empty chambers. Time spent in each chamber was measured to control for chamber bias. Following habituation, subject mice were assessed for social preference for $10 \mathrm{~min}$ by allowing interaction with Stranger 1, which was placed inside a wire cage in one chamber, and with a novel object which was placed inside an identical wire cage in the opposite chamber. Stimulus mice location was counterbalanced across trials to prevent chamber bias. Stimulus mice were conspecific C57BL/6J mice from different litters, and were age, sex, and weight matched to the subject mice and to each other.

Behavioral experiments were performed under red lighting conditions ( $<5$ lux) by a single experimenter blind to the genotype of the animals. All experiments were videorecorded by a camera (GUPPY PRO F-125B CCD, Allied Vision Technologies, GmbH, Ahrensburg, Germany) located above the arena and analyzed post-hoc using Ethovision XT 10.1 software (Noldus, Wageningen, The Netherlands). The apparatus was cleaned after each trial with $70 \%$ ethanol and then with double-distilled water.

\section{Head-post surgery}

To prepare for awake fMRI scanning, mice were implanted with MRI-compatible head-posts, as previously described $^{14}$. Briefly, mice were anesthetized with isoflurane (1.5-2.5\%), the scalp and periosteum were removed from above the surface of the skull, and a head-post was attached to the skull using dental cement (C\&B Metabond, Parkell, Brentwood, NY, United States). Mice received a subcutaneous injection containing broad-spectrum antibiotics (Cefalexin) and analgesia (Buprenorphine) during the surgery and daily for at least 2 days after the surgery.

\section{Animal imaging and analyses}

Animal imaging was performed by experimenters blind to the genotype of the animals. MRI scans were acquired with a 9.4T MRI (Bruker BioSpin GmbH, Ettlingen, Germany), using a quadrature $86 \mathrm{~mm}$ transmit-only coil and a $20 \mathrm{~mm}$ loop receive-only coil (Bruker). Raw data were reconstructed using ParaVision 5.1 (Bruker). For both structural and functional scans, data were registered to a downsampled version of the Allen Mouse Brain Connectivity (AMBC) atlas which includes anatomical annotations ${ }^{36}$ and optical density maps from anatomical tracing experiments ${ }^{37}$.

\section{Structural MRI acquisition and analysis}

Mice underwent a single anatomical scan while being anesthetized with isoflurane $(0.5-1 \%)$ using a rapid relaxation enhancement (RARE) sequence (repetition time [TR] $6000 \mathrm{~ms}$, echo time [TE] $8.8 \mathrm{~ms}$, FA $180^{\circ}$, RARE factor 16, 36 coronal slices, matrix $160 \times 160$, field of view [FOV] $16 \times 16 \mathrm{~mm}^{2}$, voxel size $100 \times 100 \times 400 \mu^{3}$ ).

To estimate the structural changes in the A350V mouse model, a deformation-based morphometry (DBM) was used by which the structural MRI scans were registered to a target using a small-displacement non-linear registration software $^{38}$ and the Jacobian determinants were computed to serve as a measure of deformation. The target was generated by averaging the anatomical scans of WT mice $(n=12)$. To allow accurate estimation of brain volume, structural scans were kept in native space and the AMBC atlas was aligned to the target. Whole-brain volume, excluding the olfactory bulb and cerebellum, was manually defined for each animal and a whole-brain template was generated by averaging the manual labels for WT mice only. We used AMBC anatomical annotations to define the regions: cerebral cortex excluding the olfactory bulb (CTX), hippocampal region (HIP), thalamus $(\mathrm{TH})$, caudoputamen (CP), and piriform cortex (PIR), and thereby allowing an estimation of anatomical changes between the groups. We further defined six cortical modules: Prefrontal, Anterolateral, Somatomotor, Visual, Medial, and Temporal based on Harris et al. ${ }^{39}$ with the exception of the rostrolateral visual area (VISrl) which is part of the Medial module and not the Visual module as originally defined.

\section{Resting-state fMRI acquisition and preprocessing}

Mice of each genotype ( $n=13$ per group) underwent multiple awake head-fixed fcMRI sessions, as previously 
described $^{14,17,40,41}$. Imaging of animals in the awake state may be impacted by stress and in group comparisons, genotype-specific response to stress may be raised as a concern. This potential concern was mitigated by acclimatizing the animals to head-fixation and the scanner environment prior to data acquisition, thereby reducing stress of the animals ${ }^{34}$ for data included in functional connectivity analyses. Further, genotype-specific differences due to stress are unlikely to have had a significant impact since anxiety-like behaviors were previously shown to be equivalent in this specific mouse model using the open field test ${ }^{28}$. For each session, BOLD contrast scans were acquired using spin echo-echo planar imaging (SE-EPI) sequence (TR $2500 \mathrm{~ms}$, TE $18.398 \mathrm{~ms}, \mathrm{FA} 90^{\circ}, 30$ coronal slices, matrix $96 \times 64$, FOV $14.4 \times 9.6 \mathrm{~mm}^{2}$, voxel size $150 \times 150 \times 450 \mu^{3}$ ) as it is more sensitive to microvasculature ${ }^{42}$, allowing for a better contrast-to-noise ratio $^{43}$. Animals were scanned for $6-8$ sessions, four runs per session and 200 repetitions per run. There were no significant differences in the number of included sessions between A350V $(6.461 \pm 1.126$ [mean \pm SD] $)$ and WT $(6.846 \pm 0.688$; unpaired $t$ test; $t(24)=1.05, p=0.304)$.

Raw data were preprocessed as previously descri$\operatorname{bed}^{14,17,40,41}$, including removal of the first two volumes for T1-equilibration effects, compensation of slice dependent time shifts, rigid body correction for head motion, registration to a downsampled version of the AMBC atlas ${ }^{36,37}$, and intensity normalization. Data scrubbing was applied as previously described ${ }^{14,44}$ with exclusion criteria of $50 \mu \mathrm{m}$ framewise displacement and derivative root-mean-square variance over voxels (DVARS) of $150 \%$ inter-quartile range (IQR) above the 75 th percentile and exclusion of one additional frame after the detected motion. Sessions with fewer than 60 included frames were excluded. The data subsequently underwent demeaning and detrending, nuisance regression of six motion axes, ventricular and white matter signals and their derivatives, temporal bandpass filtering to retain frequencies between $0.009-0.08 \mathrm{~Hz}$, and spatial smoothing with a full width at half maximum of $600 \mu \mathrm{m}$.

\section{Functional connectivity analysis}

To estimate functional connectivity, a region of interest (ROI, also termed seed) was defined (described below) and its time course was extracted. Seed-based Fisher's $z$ transformed Pearson's $r$ correlation maps $(z(r))$ were averaged across sessions to provide a subject-specific map. A quality assurance analysis was performed to estimate functional connectivity specificity in the WT cohort, as previously shown ${ }^{45}$. This analysis reveals a bias (i.e., values fall below the diagonal) toward the specific interhemispheric connection in all mice, and 9/13 mice meet a more conservative criterion for the definition of specific functional connectivity (Fig. S1), justifying the inclusion of all animals scanned with the above parameters. For group-level analyses, the subjectspecific maps were submitted to a one-sample $t$ test for each animal group separately or a two-sample $t$ test when comparing the groups to each other (SPM, Wellcome Department of Cognitive Neurology, London, UK). In addition, seed-to-seed correlations were calculated and averaged across sessions to estimate functional network alterations between two defined regions and to further examine whether functional connectivity was correlated with each animal's behavior.

\section{Structure-function analysis}

To estimate brain-wide alterations in the A350V mouse model in a data-driven approach, the overlap between functional and anatomical connectivity was tested by comparing the optical density maps with the fcMRI maps, as elaborately described previously ${ }^{14}$ (see also refs. ${ }^{13}$ and ${ }^{46}$ ) with modifications that allow for intergroup comparison (see Fig. S2 and Supplementary Materials for analysis validation). This analysis approach allows testing the hypothesis that functional alterations in the mutant group will result in altered structure-function relations. An advantage of this specific analysis approach is that it is not threshold dependent. For intergroup analysis, correlation maps $(z(r))$ of individual animals ( $n=13$ per group) were used to estimate anatomical prediction of functional connectivity in the six cortical modules described previously ${ }^{39}$. Functional connectivity was derived from the A350V and WT acquired here while anatomical connectivity was derived from C57BL/6J animals who were used to construct the AMBC atlas $^{37}$. Correlation maps $(z(r))$ were extracted based on seeds (a seven voxels three-dimensional cross) which corresponds to 101 out of the $122 \mathrm{C} 57 \mathrm{BL} / 6 \mathrm{~J}$ injection sites that were used to define the six cortical modules; ${ }^{39}$ missing injections include 18 out of 35 injections in VISp, one in MOp and one in MOs. The location of the seeds was defined at the center of the injection site. To characterize structure-function relations we conducted a series of receiver operating characteristic (ROC) analyses as previously described ${ }^{14}$. Briefly, anatomical projection volumes were taken from $\mathrm{Oh}$ et $\mathrm{al}^{37}$ and compared to functional volume distributions over 265 statistical thresholds. A binary volume threshold of $0.05 \mathrm{~mm}^{3}$ was used to define true anatomical connections to examine the sensitivity and specificity of the prediction of anatomical connections based on functional connections. To estimate the accuracy of prediction, we calculated the area under each ROC (auROC) curve, which estimates the relationship between true-positive and false-positive predictions at different thresholds, indicating how well functional connectivity discriminates between anatomically connected and unconnected regions.

To further examine the functional alterations of regions with altered structure-function relations, a Sørensen-Dice 
similarity coefficient was calculated $(D=2 \times(\mathrm{A} \cap \mathrm{B}) /(|\mathrm{A}|$ $+|\mathrm{B}|))$ and used to quantify the overlap between two tracer injections within the same cortical region such that overlapping projection maps will not be considered separately. Finally, a seed-based analysis was computed to further assess intergroup differences using unpaired two-sample Student's $t$-test.

\section{Statistical analyses}

All data were analyzed using MATLAB R2018a (The Mathworks, Natick, MA, USA) except for behavioral data in which analysis of variance (ANOVA) was analyzed using jamovi 1.0.7 ${ }^{47}$. Sample size was estimated based on previous studies ${ }^{17,20,40}$. The Lilliefors test was used to determine normality. Behavioral data were found to be normally distributed. As for the MRI data, since part of the data were not normally distributed, group comparisons were performed using the non-parametric statistics Mann-Whitney $U$-test. Correspondence between manual and automated volumetric measurements and correlation between fcMRI alteration and social behavior were evaluated using Pearson's $r$ correlation score. The effect size in the non-parametric statistics was measured as $r=z / \sqrt{ } n$. Structural analyses were corrected for multiple comparisons using the false discovery rate (FDR) procedure ${ }^{48}$. The analysis approach was to use the fcMRI structure-function data as a discovery sample for seed selection. Next, auROC values were compared using two-tailed Mann-Whitney $U$-test and only ROI that showed differences were further analyzed (defined using a critical $U$ value of $\alpha=0.05$ for sample sizes of $n_{1}=13$ and $n_{2}=13$ ). ROIs identified with this approach were used to compute an fcMRI contrast map between A350V and WT and were corrected for multiple comparisons, first using cluster-level correction and second using Bonferroni correction to account for the number of network comparisons $\left(p<0.05, n_{\text {networks }}=4\right.$, one-way comparison based on auROC findings). Only a single ROI and a single region survived this correction, and it was subsequently correlated with behavior. Intergroup differences of the behavioral data were quantified using two-way repeated-measures ANOVA, with side preference (i.e., time spent in close interaction with Stranger 1/object) defined as a repeated measure factor and genotype as between-subject factor. Detection of outliers in the data was conducted following the steps suggested in $\mathrm{Seo}^{49}$. Symmetry and normality assumptions were assessed for selecting the appropriate detection method. Hence, Tukey's and median absolute deviation methods were used to detect and exclude outliers from the behavioral data.

\section{Data sharing}

All imaging raw data and the relevant codes used in this study are available in BIDS format on OpenNeuro, https://openneuro.org/datasets/ds003468.

\section{Results}

Brain morphology alterations in A350V mouse model

Structural alterations have been reported in both humans with ASD and mouse models of $\mathrm{ASD}^{50,51}$. Therefore, we sought to examine whether there are structural volumetric differences in the brains of A350V mice relative to WT littermates by using manual segmentation and automatic non-linear registration of structural MRI. Manual quantification of volumes $(n=$ 13 per group) showed a statistically significant increase in whole-brain volume in A350V mice as compared to WT littermates (Fig. 2a; Mann-Whitney $U$-test, $U=41$, $p=0.027, r=0.432$ ), but no difference in ventricle volume (WT: $9.937 \pm 0.418 \mathrm{~mm}^{3}[$ mean \pm SEM]; A350V: $9.685 \pm 0.281 \mathrm{~mm}^{3} ; U=78, p=0.758$ ). After subtraction of the ventricle volume, the intracranial volume of the A350V mice $\left(359.912 \pm 3.405 \mathrm{~mm}^{3}\right)$ was still found to be significantly larger relative to WT littermates $\left(348.918 \pm 2.484 \mathrm{~mm}^{3} ; U=41, p=0.027, r=\right.$ 0.432), suggesting that the increased whole-brain volume is unlikely to be due to hydrocephalus. Next, we used a deformation-based morphometry method ${ }^{38}$ to automatically evaluate changes in the volumes of the entire brain and substructures. Consistent with the manual quantification, whole-brain volume as expressed by the Jacobian deformation was significantly larger in A350V compared to WT (Fig. 2a; $U=33, p=0.009$, $r=0.512$ ). In addition, a significant correlation between the manual whole-brain volume measurements and the Jacobian deformation measure (Fig. $2 \mathrm{~b} ; n=13, r(24)=$ $0.845, p<0.001)$ suggested that the automatic deformation measure with the current structural imaging acquisition parameters was reliable. The AMBC atlas was used to define the substructures of the brain and compare volumes of A350V to those of WT (Fig. 2c). We found increased volumes in A350V relative to WT in the cerebral cortex without the olfactory bulb $(U=$ 22, $p=0.007$; FDR-corrected, $r=0.623)$ and the hippocampal region $(U=31, p=0.016$; FDR-corrected, $r=0.533$ ), but no significant volume differences in the thalamus, caudoputamen, and piriform cortex ( $U$ values > $42, p$ values $>0.052$; FDR-corrected).

Next, we tested whether the observed structural alterations are attributed to specific regions of the cortex by dividing it into six modules ${ }^{39}$ (Prefrontal, Anterolateral, Somatomotor, Visual, Medial, and Temporal; Fig. 2d). Volumes were significantly larger in Prefrontal $(U=30$, $p=0.017 ; \quad$ FDR-corrected, $\quad r=0.543), \quad$ Somatomotor ( $U=30, p<0.017$; FDR-corrected, $r=0.543$ ), Anterolateral $(U=39, p=0.031$; FDR-corrected, $r=0.452)$, and Medial $(U=35, p=0.024$; FDR-corrected, $r=0.492)$ modules in the A350V compared to WT, with no significant differences observed in the Visual $(U=67, p=0.383)$ and Temporal $(U=69, p=0.441)$ modules. 

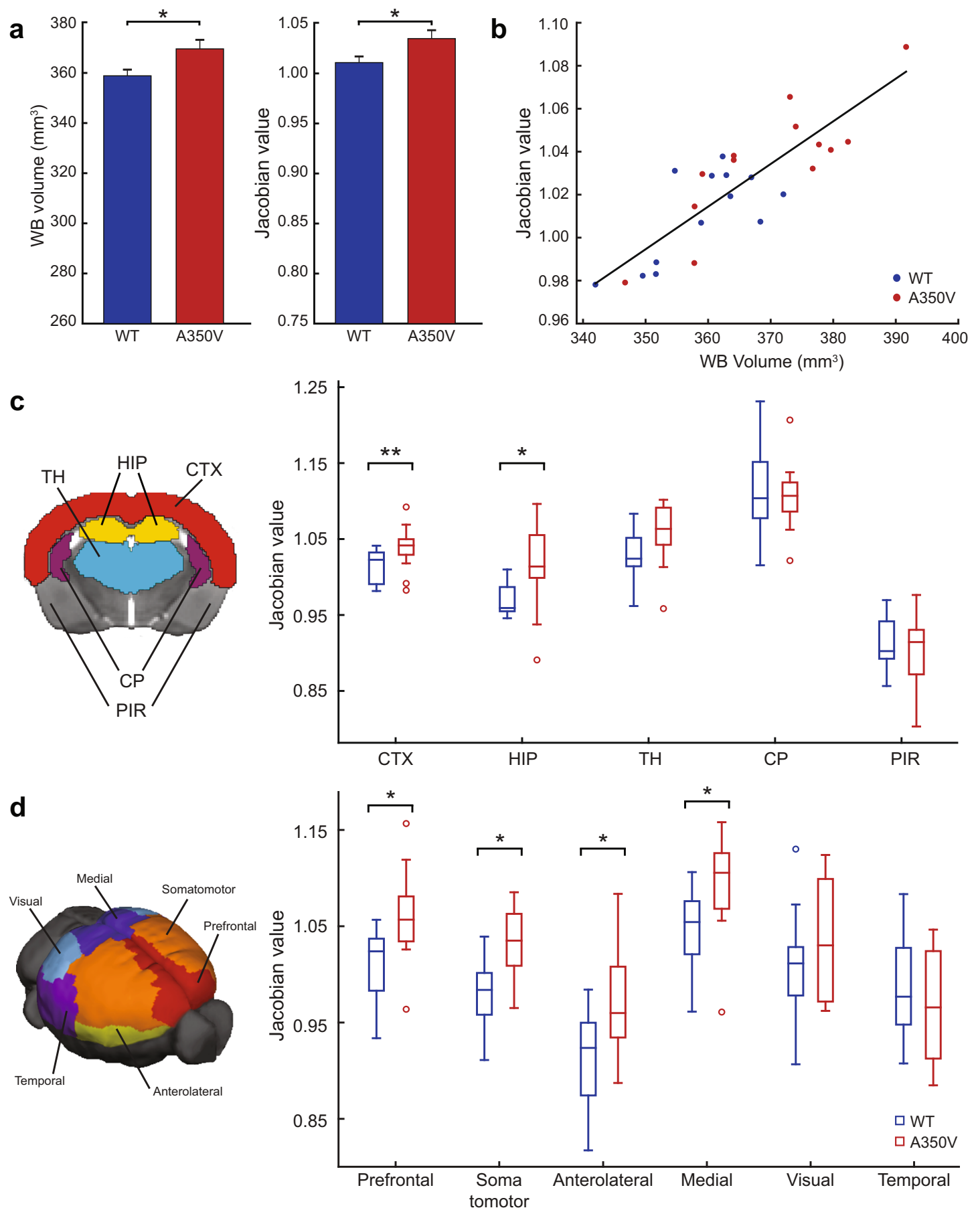

Fig. 2 Structural alterations in A350V IQSEC2 mouse model. a Whole-brain volume increase in A350V mice shown by manual volume quantification (left) and automatic registration evaluation (right). b Correlation between Jacobian determinant and whole-brain volume showing an agreement between the two evaluation methods. $\mathbf{c}$ Jacobian determinant of the different brain structures depicted on the left indicates increased volume of CTX and HIP in A350V mice. $\mathbf{d}$ Jacobian determinant of the six cortical modules depicted on the left indicates increased volume of the Prefrontal, Somatomotor, Anterolateral, and Medial modules in A350V mice. Data indicate mean \pm SEM. Boxplots represent the median (center line), interquartile range (box limits), extreme data points (whiskers), and outlier (circles). Mann-Whitney U-test; ${ }^{*} p<0.05,{ }^{* *} p<0.01$, FDR-corrected. CTX cerebral cortex, HIP hippocampal region, TH thalamus, CP caudoputamen, PIR piriform area. 


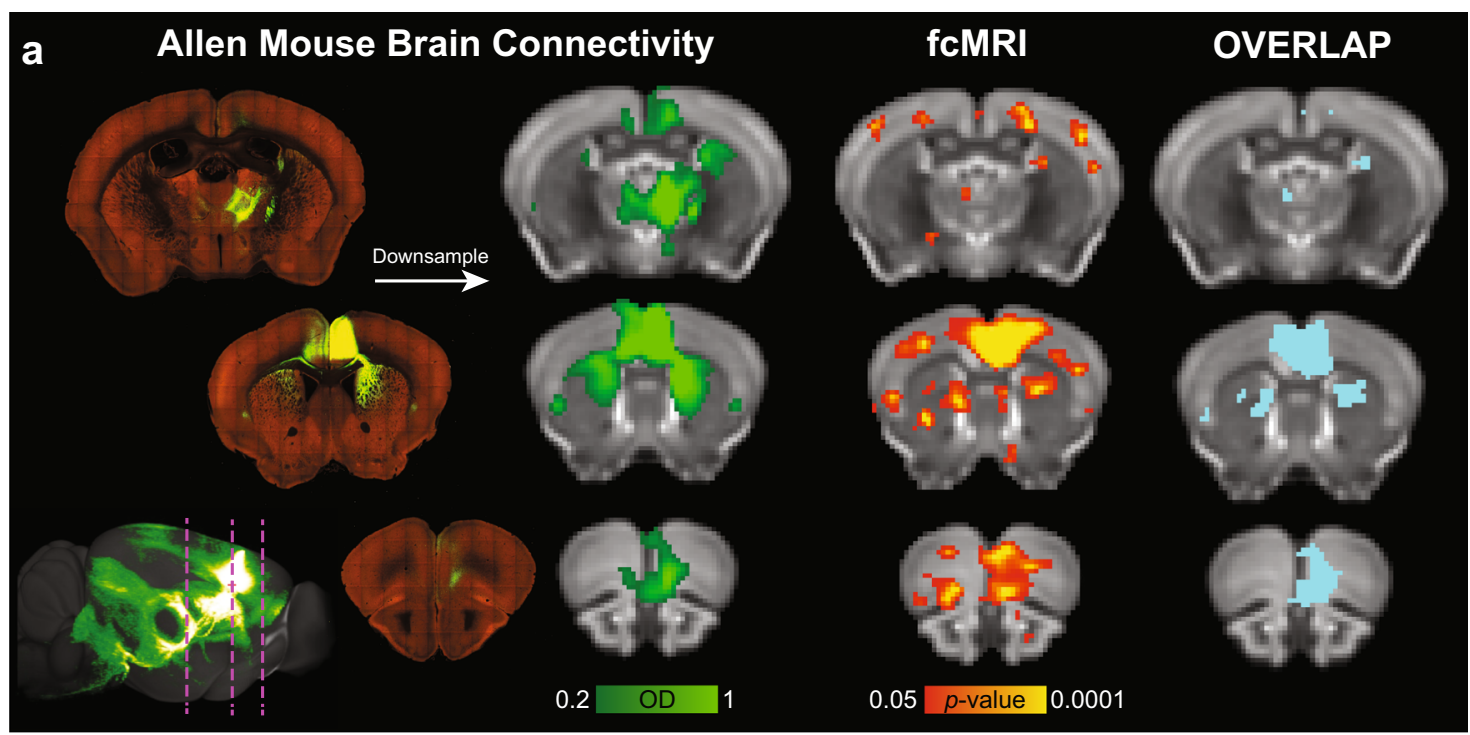

b

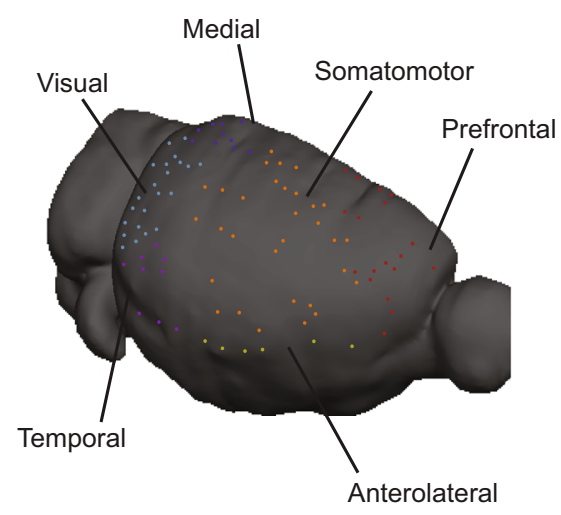

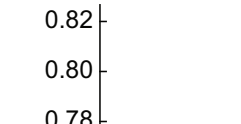

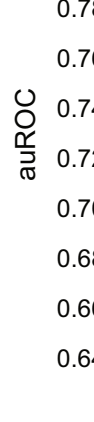

0.64

Prefrontal<smiles>[SiH3]</smiles><smiles>C1CC[AlH]C1</smiles>
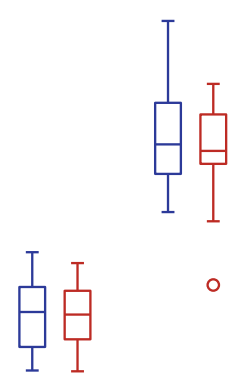

o

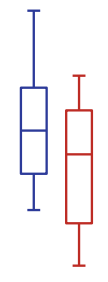

口 WT

$\square$ A350V

Somato

motor

Fig. 3 Structure-function relations of the six cortical modules. a Analysis scheme for comparing structure-function relations using MRI shown for Allen mouse brain connectivity (AMBC) atlas experiment \#112514202, ACAv injection. Three representative coronal slices showing multiple anatomical connections (top, left) in AMBC space and a maximum intensity projection sagittal view of signal density (bottom, left). Approximate locations of the presented coronal sections are indicated by magenta dashed lines. The arrow indicates the corresponding downsampled coronal slices (optical density, [OD] in green-light green, normalized OD > 0.2). An fcMRI statistical parametric map (in red-yellow) of positive correlations of ACAv on a downsampled version of the AMBC Atlas that matches the original fMRI resolution; $p<0.05$, corrected for multiple comparisons using family-wise error rate correction for the whole mouse brain. Overlap of functional and anatomical connectivity (pale blue) demonstrates a close agreement across the two modalities. $\mathbf{b}$ The seeds corresponding to AMBC tracer injections are illustrated on a mouse cortical surface. Seeds are color coded for the six cortical modules. c The six cortical modules auROC values demonstrate equivalent structure-function relations between the two groups for all modules. $n=13$ per group; Boxplots represent the median (center line), interquartile range (box limits), extreme data points (whiskers), and outlier (circles).

\section{Structure-function analysis reveals cortical alterations in A350V mice}

To assess the functional disruption in the A350V mouse brain, we used functional connectivity MRI. It has been previously shown that fcMRI tracks anatomical connectivity, and that this coupling can be used to reveal functional connectivity based on brain architecture ${ }^{13,14,46,52}$. We used ROC analysis to evaluate the structure-function relations of cortical systems, by comparing the optical density tracer injection maps of the AMBC to the functional connectivity maps acquired using fcMRI (Fig. 3a). In this analysis, the AMBC anatomical connectivity serves as baseline data and is compared with multiple statistical thresholds of the functional connectivity data, allowing to evaluate the agreement between anatomical and functional connectivity. Thus, changes in functional connectivity in A350V relative to WT can lead to both an increased (closer agreement) or decreased consistency with WT structural connectivity. Since structural connectivity is derived from WT (i.e., $\mathrm{AMBC}$ ) in both groups, these changes serve to 

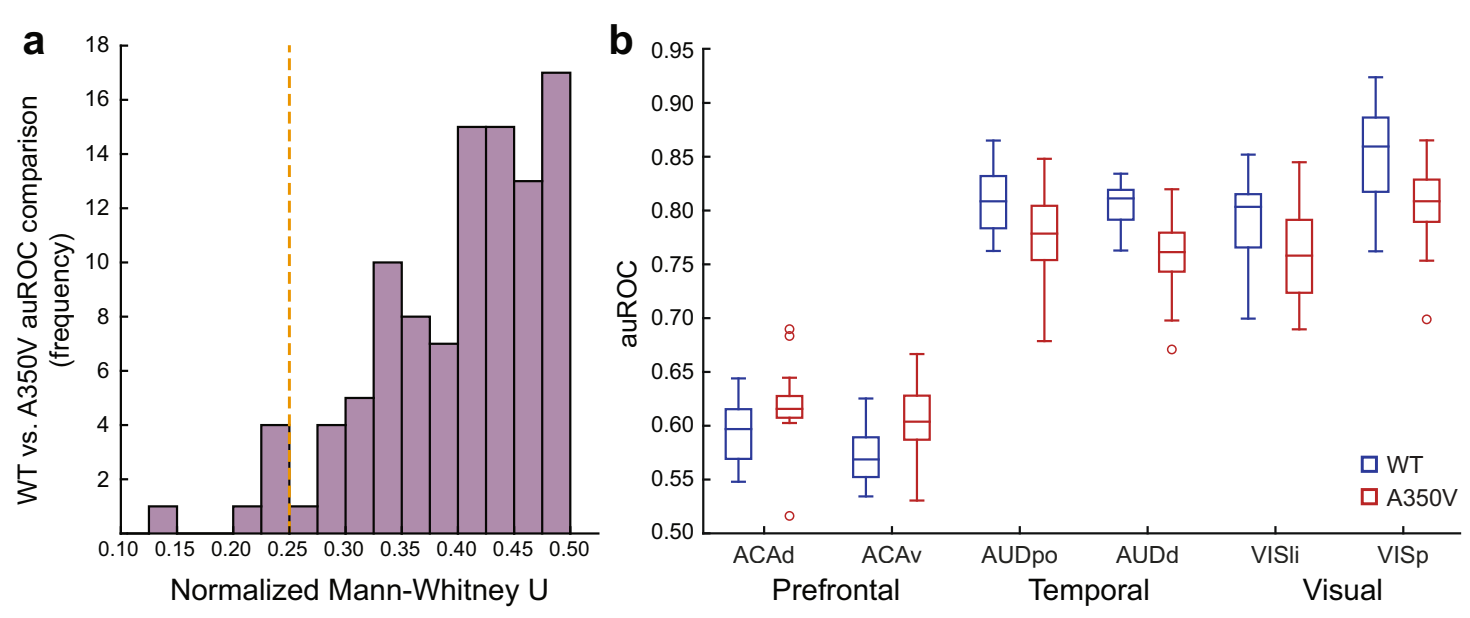

Fig. 4 Structure-function relations reveal potential altered functional connectivity in A350V relative to WT and serves as a seed selection method. a Distribution of the normalized Mann-Whitney $U$ obtained from the comparison of the 101 cortical modules seeds between WT and A350V mice. Orange dashed line indicates the six comparisons that passed the critical normalized $U$ value for sample size of $n_{W T}=13$ and $n_{\text {A350V }}=13$. $U$ values were normalized to the expected maximal $U$ value. $\mathbf{b}$ The area under receiver operating characteristic (auROC) curve values indicate closer agreement of structure-function relations in A350V mice (red) for ACAd and ACAv, and closer agreement in WT mice (blue) for AUDpo, AUDd, VISli, and VISp. Boxplots represent the median (center line), interquartile range (box limits), extreme data points (whiskers), and outlier (circles). ACAd anterior cingulate area, dorsal part, ACAv anterior cingulate area, ventral part, AUDpo posterior auditory area, AUDd dorsal auditory area, VISli laterointermediate visual area, VISp primary visual area.

identify where the A350V mutation altered intact functional connectivity. In this study, we adjusted the analysis (previously used in ref. ${ }^{14}$ ) to the individual level to enable a comparison between groups and used this thresholdindependent analysis as a data-driven approach that enables seed selection for further analyses.

Quantification of structure-function relations allows an assessment as to whether disruption in functional connectivity at the single animal level localizes to subnetworks within modules as defined by specific tracer injections and their first synapse projections. We generated seeds that correspond to the locations of 101 C57BL/6J tracer injections, computed the seed-based correlation maps of individual animals, and compared the auROC curve values, a measure of agreement between structural and functional connectivity, across the two groups. We first sought to test whether the observed anatomical macrocephaly we found in A350V mice would be reflected in the structure-function relations of the six cortical modules (Fig. 3b). When comparing the auROC values of each module between the groups by averaging all module-related seeds per animal, we found no significant differences (Fig. 3c; Mann-Whitney $U$-test, $\quad U_{\text {Prefrontal }}=77, \quad p=0.719$; $U_{\text {Somatomotor }}=83, \quad p=0.959 ; \quad U_{\text {Anterolateral }}=68, \quad p=$ $0.412 ; \quad U_{\text {Medial }}=84, p=1.00 ; \quad U_{\text {Visual }}=76, p=0.681$; and $U_{\text {Temporal }}=55, p=0.137$ ).

Next, to identify potentially specific disrupted regions we quantified structure-function relations for individual injections $^{39}$. Namely, individual seed regions which will be directly contrasted between the groups will be subsequently selected for further analyses using a threshold independent approach. We discovered differences in six injections located in the anterior cingulate, auditory and visual cortices (Fig. 4). The auROC values for the ACAd and ACAv regions were higher in $\mathrm{A} 350 \mathrm{~V}$ relative to $\mathrm{WT}$ $\left(U_{\mathrm{ACAd}}=42, p=0.031, r=0.422 ; U_{\mathrm{ACAv}}=34, p=0.01\right.$, $r=0.503)$, suggesting a closer agreement between the anatomical connectivity and the functional connectivity in A350V. Conversely, AUDpo, AUDd, VISli, and VISp regions showed reduced auROC values in $\mathrm{A} 350 \mathrm{~V}$ relative to WT $\left(U_{\text {AUDpo }}=42, p=0.031, r=0.422 ; U_{\text {AUDd }}=24\right.$, $p=0.002, \quad r=0.603 ; \quad U_{\mathrm{VISli}}=42, \quad p=0.031, \quad r=0.422$; $\left.U_{\mathrm{VISp}}=42, p=0.031, r=0.422\right)$.

Following the altered structure-function relations described above, we sought to further investigate whether these regions demonstrated functional changes at the network level. Since the pairs of the regions (ACAd/ACAv, AUDpo/AUDd, and VISli/VISp) are potentially overlapping to the extent that they are effectively identical, we evaluated the overlap of the tracer injections for each pair of proximal regions using the Sørensen-Dice similarity coefficient. We found a substantial overlap for ACAd/ACAv and AUDpo/ AUDd (Dice index of 0.861 and 0.757 , respectively), and therefore, in further analyses, they were considered as single regions, designated $\mathrm{ACAd} / \mathrm{v}$ and $\mathrm{AUDpo} / \mathrm{d}$. The VISli and VISp similarity coefficient was 0.545 


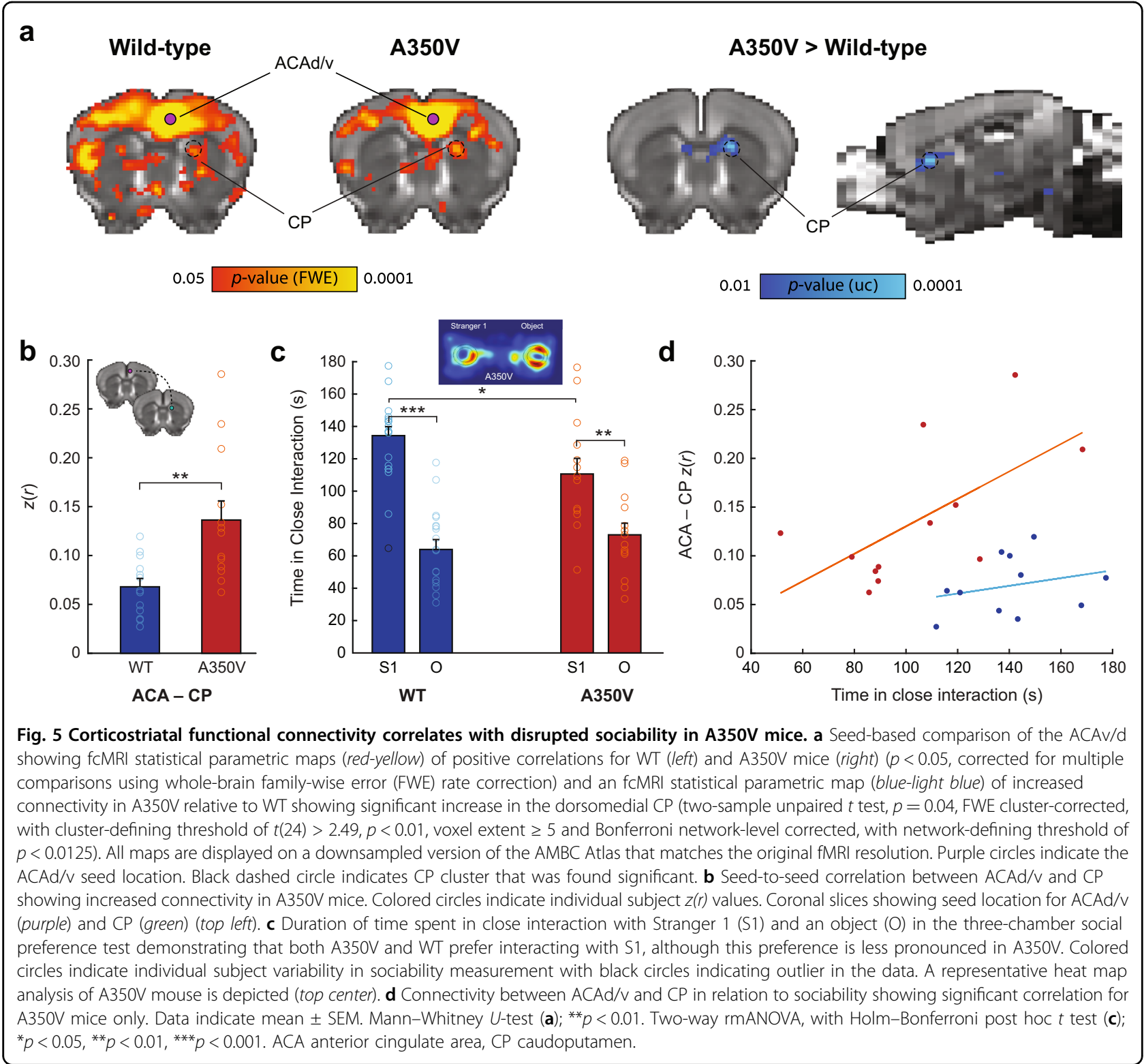

and they were therefore considered as two separate networks.

\section{Corticostriatal alterations in functional connectivity are associated with social impairments}

To further dissect the alterations found in the structure-function analysis we computed a two-sample $t$ test statistical parametric map between the groups for ACAd/v, AUDpo/d, VISli, and VISp seed-based correlation maps. Seed-based correlation maps were averaged across sessions to provide a subject-specific map resulting in 13 maps for A350V and 13 maps for WT for each of the above four seed regions. Of the above regions, only the comparison between A350V and WT of the ACAd/v seed-based maps showed a significant increase in functional connectivity in A350V mice which was exclusive to the dorsomedial caudoputamen (CP; Fig. $5 \mathrm{a} ; t$ test, $p=0.04$, FWE cluster-level corrected, with clusterdefining threshold of $t(24)>2.49, p<0.01$ and Bonferroni network-level corrected, with network-defining threshold of $p<0.0125)$. Next, we performed a seed-toseed correlation analysis between the ACAd/v and the $\mathrm{ROI}$ in the CP that was found in the prior analysis, obtaining correlation values at the individual animal level (Fig. 5b). As expected, A350V mice displayed increased ACAd $/ \mathrm{v}-\mathrm{CP}$ connectivity relative to WT $(U=26$, $p=0.002, r=0.583$ ).

In the human index case with the A350V IQSEC2 mutation abnormalities in social behaviors were found ${ }^{32}$. Thus, social behavior was assessed using a three-chamber 
sociability test (Fig. 5c). When we examined social preference as a measure of time spent in close interaction, a repeated-measures ANOVA revealed a significant main effect of Side $\left(\mathrm{F}(1,29)=45.39, p<0.001, \eta_{p}{ }^{2}=0.61\right)$, as well as an interaction between Side and Genotype $(\mathrm{F}(1,29)=$ 4.17, $p=0.05, \eta_{p}{ }^{2}=0.126$ ). A further post-hoc analysis revealed a significant difference in time spent in close interaction with an unfamiliar mouse between $\mathrm{A} 350 \mathrm{~V}$ and WT ( $t$ test, $t=2.444, p=0.035$, Holm-Bonferroni correction). Collectively, these results indicate that both A350V and WT mice prefer interacting with an unfamiliar mouse over an object, yet this preference was reduced in A350V relative to WT.

Finally, to test whether the abnormal social behavior found in A350V model can be explained by the neurophysiological alterations that were obtained from the same animal cohort, we correlated the social preference measurement both with the structural deformation and the seed-to-seed functional connectivity. The observed volumetric differences showed no significant correlation with the behavioral impairments that were found (data not shown). When examining the relations between the altered ACAd/v-CP functional connectivity with the observed social behavior, we found a significant correlation of functional connectivity magnitude and social preference for A350V only (Fig. 5d; A350V: Pearson's $r(10)=0.627, p=0.029$; WT: Pearson's $r(9)=0.267, p=0.426)$. Namely, equivalent functional connectivity between $\mathrm{A} 350 \mathrm{~V}$ and WT is correlated with a reduction in social interaction in A350V, while increased corticostriatal connectivity in A350V is associated with normal levels of social interaction as found in WT.

\section{Discussion}

We explored the neurophysiological changes in a novel transgenic A350V mouse model which is based on a human de novo mutation in the IQSEC2 gene associated with ASD. We show that A350V male mice exhibit overall increased brain volume, restricted to the cerebral cortex and hippocampus, along with indication for alterations in structure-function relations of the frontal, auditory, and visual systems. Leveraging this data-driven approach, we found changes in corticostriatal functional connectivity which were associated with individual variability in social behavior, demonstrating that the equivalent levels of corticostriatal functional connectivity between A350V and WT correspond to reduced social interaction in the three-chamber social preference test in A350V. Collectively, these findings suggest that the A350V missense mutation in the Iqsec2 gene induces ASD features that are derived from corticostriatal dysfunction and may serve as a translational model from human to mouse.
IQSEC2 is one of many ASD-linked genes that encode postsynaptic density proteins of glutamatergic synapses, such as CNTNAP (contactin-associated protein), neuroligin, NF1 (neurofibromin 1), SHANK family, and more ${ }^{53}$. It has been previously proposed that the IQSEC2 protein plays a role in synaptic plasticity and dendritic spine formation $^{24,27,54}$. A recent study showed that changes in Iqsec 2 dosage are linked with alterations in neural morphology and specifically in dendritic spines ${ }^{54}$. While the signal transduction pathway of IQSEC2 is not fully understood, it has been previously suggested that IQSEC2 functions as a guanine nucleotide exchange factor (GEF) for ADP-ribosylation factors (Arfs), and that the Arf-GEF activity further regulates AMPA receptor trafficking ${ }^{26,27}$. In the case of the A350V mutation, it has been proposed that there is a constitutive activation of the GEF activity of IQSEC2 that results in increased endocytosis of AMPA receptors via constant activation of Arf6 along with other processes that are less understood ${ }^{28}$.

When addressing the macroscopic changes reported here at the cellular level, there are several possible explanations for the observed changes, and in particular the increase in corticostriatal functional connectivity. An important finding that may explain IQSEC2-mediated changes in AMPA receptors at the circuit level has recently been reported by Sah and colleagues ${ }^{55}$ who demonstrated that Iqsec2 is expressed not only in excitatory glutamatergic neurons but also in GABAergic interneurons. This group then further demonstrated in an Iqsec 2 knockout mouse model that the loss of Iqsec2 specifically resulted in increased activity of these inhibitory interneurons. They hypothesize that this increased activity of the GABAergic neurons in the Iqsec2 knockout model was due to an upregulation of AMPA receptors in these cells. Consequently, there are several ways in which such an observation could explain increases or decreases in functional connectivity. The A350V model appears to be a gain of function model and is associated with constitutive activation of Iqsec $2^{28}$, which may produce the opposite effect as seen in a knockout model on these GABAergic neurons and AMPA receptors ${ }^{27,28,56}$. Moreover, the precise impact of the A350V mutation on glutamatergic neurons projecting from the cortex to the striatum or on the medium spiny neurons of the striatum is unknown, limiting the ability to pinpoint the mechanism driving the observed effect. To fully understand the impact of A350V, future studies will need to explore the contribution of these specific cell types to the observed change in functional connectivity and whether they impact social behavior.

In ASD, human studies have consistently demonstrated a modest but significant increase in overall brain volume in toddlers ${ }^{57}$. In a recent study characterizing structural alterations in 26 ASD mouse models using MRI, these 
models were classified into three subtypes according to alterations in volume and connectivity ${ }^{50}$. The authors found that all of the mouse models that were associated with increased brain volume were classified in the same subtype (NRXN1 $1 \alpha^{-1-}, \mathrm{NRXN} 1 \alpha^{-/+}$and FMR1 $1^{-/ y} \mathrm{FVB}$ ). However, other models within this subtype either showed no change in brain volume (En2 $2^{-/-}$and FMR1 $\left.{ }^{-/ y} \mathrm{~B}^{\wedge}\right)$ or decrease in brain volume $\left(\right.$ SHANK $^{-1-}$ and SHANK3 $^{-1+}$ ). Collectively, this evidence suggests that brain volume is not a critical distinguishing feature. Related to the reported A350V mutation, in a recent study using female mice ${ }^{58}$, heterozygous loss of function of the Iqsec2 gene showed thinning of the corpus callosum along with increased volume of the hippocampus and specifically the dentate gyrus within it (cortical volume was not reported). Here, male $\mathrm{A} 350 \mathrm{~V}$ mice presented an increase in whole-brain volume that was found to derive from cortical and hippocampal enlargement. We found a volume increase in four out of the six cortical modules, suggesting a nonspecific cortical enlargement. Further, unlike the functional connectivity results, increased volume was not correlated with the altered social behavior identified in this model and is possibly relevant to other behavioral features presented in this model ${ }^{28}$. Taken together with Ellegood et al. ${ }^{50}$, it is suggested that the observed macrocephaly in A350V mice is unlikely to be involved in the pathophysiology of the brain connectivity we report here.

Numerous studies implicated corticostriatal alterations in ASD pathophysiology in humans and animals. A study comparing ASD and typically developing children showed increased functional connectivity between striatal subregions and both the association and limbic cortices ${ }^{59}$. In a study using connectivity-based parcellation, a difference in the organization of corticostriatal circuitry in ASD was reported, demonstrating that the separation of the putamen to distinct anterior and posterior clusters is absent in ASD participants, and is potentially driven from a connectivity fingerprint of several cortical regions ${ }^{60}$. Previous studies in the SHANK3 mouse model have demonstrated dysfunction of corticostriatal activity ${ }^{19,61}$, specifically by recording AMPA receptor-mediated currents in spiny projection neurons of dorsomedial striatum ${ }^{19}$, with the striatum playing a causal role in repetitive grooming behavior $^{62}$. A recent fcMRI study in the same mouse model showed an overall prefrontal hypoconnectivity along with disrupted functional connectivity between the ACA and the caudoputamen ${ }^{21}$. Consistent with these reports, we found altered functional connectivity between the ACA and the dorsomedial striatum. Collectively, these findings emphasize the contribution of association and limbic corticostriatal hyper- or hypo-connectivity to ASDrelated pathology. Moreover, anatomical homology and recapitulation of findings when using fMRI in ASD genetic mouse models suggest that the pathophysiological findings in genetic animal models are likely to translate to humans.

Studying the contribution of specific brain structures to behavior is particularly important when it comes to understanding neuropathology of diseases, as it can help dissect the relevance of specific brain structures to behavioral features and subsequently identify disease pathophysiology. In a task-based fMRI study, hyperconnectivity between the anterior cingulate cortex and the caudate has been shown to be associated with deactivation to social rewards in ASD participants ${ }^{63}$. Several studies of ASD mouse models linked brain function and social behavior impairments. Homozygous loss of the CNTNAP2 (contactin-associated protein-like 2) gene results in fronto-posterior hypoconnectivity, a finding that has been reported in several ASD models, which was further associated with reduced social investigation in a male-female social interaction test ${ }^{20}$. In addition to ACA-CP disrupted connectivity, the SHANK3 fcMRI study mentioned above showed that prefrontal hypoconnectivity is correlated with socio-communicative deficits $^{21}$. These findings are consistent with a study showing that specific deletion of SHANK3 in the ACA is sufficient to induce social impairments, which can be improved by pharmacological enhancement of AMPA receptors, suggesting that the ACA plays a role in regulating social behavior $^{64}$. In our study, A350V mice presented high variability in the sociability measurement using the threechamber sociability task. We found this variability to be correlated with the connectivity levels between the ACA and the dorsomedial striatum. While the A350V mouse model showed an overall increase in corticostriatal connectivity, we observed that the A350V mice with functional connectivity levels equivalent to WT littermates presented low sociability levels as compared to these WT littermates, while the A350V mice with high connectivity levels presented normal sociability levels. This result suggests that increased levels of connectivity may reflect a compensatory neural response mitigating social impairments in the A350V model. The concept of the neural response of compensatory nature on cognitive function has long been recognized ${ }^{65,66}$ and has been previously suggested to underly variability in disease severity in $\mathrm{ASD}^{67}$. To fully understand the pathophysiology of the A350V model future studies will need to causally address the role of ACA in social impairments. Taken together, we can only speculate that the underlying cellular mechanism explaining functional network compensation may involve heterogeneity in AMPA receptors expression levels within the ACA both in glutamatergic and GABAergic neurons ${ }^{55}$.

Finally, we would like to propose the A350V Iqsec2 mouse as a putative ASD model. Overall, our results are consistent with brain alterations found in several ASD 
models. The diversity of genetic mutations which lead to similar brain-wide and associated behavioral changes in ASD suggests that the ability to offer precise therapies for ASD depends on understanding how diverse neural pathologies are linked to ASD. Specifically, future research which will more fully characterize the cellular pathophysiological changes that drive the brain-wide level effects observed in the A350V model, and the relation of these changes to the brain-wide changes observed across the diverse ASD models, will facilitate progress in understanding the causes of ASD in A350V IQSEC2 and in proposing paths to therapy.

\section{Acknowledgements}

This work was supported by the Israel Science Foundation (770/17; to I.K.), the National Institutes of Health (1R01NS091037; to I.K.), and the Adelis Foundation (to I.K.). We thank Technion's Biological Core Facilities and Edith Suss-Toby for her assistance with MRI, and the Technion Preclinical Research Authority for assistance with animal care.

\section{Author contributions}

D.L., I.K., N.S.L. and A.P.L. designed the study. D.L., A.K. and N.C. collected the data. D.L. and E.B. analyzed the data. D.L. wrote the first draft and all authors assisted in writing and reviewing the paper.

\section{Conflict of interest}

The authors declare no competing interests.

\section{Publisher's note}

Springer Nature remains neutral with regard to jurisdictional claims in published maps and institutional affliations.

Supplementary information The online version contains supplementary material available at https://doi.org/10.1038/s41398-021-01289-8.

Received: 9 September 2020 Revised: 15 February 2021 Accepted: 24 February 2021

Published online: 22 March 2021

\section{References}

1. Geschwind, D. H. \& State, M. W. Gene hunting in autism spectrum disorder: on the path to precision medicine. Lancet Neurol. 14, 1109-1120 (2015).

2. Takata, A. et al. Integrative analyses of de novo mutations provide deeper biological insights into autism spectrum disorder. Cell Rep. 22, 734-747 (2018).

3. Elsabbagh, M. et al. Global prevalence of autism and other pervasive developmental disorders. Autism Res. 5, 160-179 (2012).

4. Mannion, A. \& Leader, G. Comorbidity in autism spectrum disorder: A literature review. Res. Autism Spectr. Disord. 7, 1595-1616 (2013).

5. Matson, J. L. \& Nebel-Schwalm, M. S. Comorbid psychopathology with autism spectrum disorder in children: An ovenview. Res. Dev. Disabil. 28, 341-352 (2007).

6. Gilman, S. R. et al. Rare de novo variants associated with autism implicate a large functional network of genes involved in formation and function of synapses. Neuron 70, 898-907 (2011).

7. Parikshak, N. N. et al. Integrative functional genomic analyses implicate specific molecular pathways and circuits in autism. Cell 155, 1008-1021 (2013).

8. Vasa, R. A., Mostofsky, S. H. \& Ewen, J. B. The disrupted connectivity hypothesis of autism spectrum disorders: time for the next phase in research. Biol. Psychiatry Cogn. Neurosci. Neuroimaging 1, 245-252 (2016).

9. Liska, A. \& Gozzi, A. Can mouse imaging studies bring order to autism connectivity chaos? Front. Neurosci. 10, 484 (2016).

10. Fox, M. D. \& Raichle, M. E. Spontaneous fluctuations in brain activity observed with functional magnetic resonance imaging. Nat. Rev. Neurosci. 8, 700-711 (2007).

11. Power, J. D., Schlaggar, B. L. \& Petersen, S. E. Studying brain organization via spontaneous fMRI signal. Neuron 84, 681-696 (2014).
12. Zerbi, V., Grandjean, J., Rudin, M. \& Wenderoth, N. Mapping the mouse brain with rs-fMRl: An optimized pipeline for functional network identification. Neuroimage 123, 11-21 (2015).

13. Stafford, J. M. et al. Large-scale topology and the default mode network in the mouse connectome. Proc. Natl Acad. Sci. USA 111, 18745-18750 (2014).

14. Bergmann, E., Zur, G., Bershadsky, G. \& Kahn, I. The organization of mouse and human cortico-hippocampal networks estimated by intrinsic functional connectivity. Cereb. Cortex 26, 4497-4512 (2016).

15. Buckner, R. L. et al. Cortical hubs revealed by intrinsic functional connectivity: mapping, assessment of stability, and relation to Alzheimer's disease. J. Neurosci. 29, 1860-1873 (2009).

16. Bertero, A. et al. Autism-associated 16p11.2 microdeletion impairs prefrontal functional connectivity in mouse and human. Brain 141, 2055-2065 (2018).

17. Shofty, B. et al. Autism-associated Nf1 deficiency disrupts corticocortical and corticostriatal functional connectivity in human and mouse. Neurobiol. Dis. 130, 104479 (2019).

18. Scott-Van Zeeland, A. A. et al. Altered functional connectivity in frontal lobe circuits is associated with variation in the autism risk gene CNTNAP2. Sci. Transl. Med. 2, 56 ra80 (2010).

19. Peixoto, R. T. et al. Early hyperactivity and precocious maturation of corticostriatal circuits in Shank3B ${ }^{-1-}$ mice. Nat. Neurosci. 19, 716-724 (2016).

20. Liska, A. et al. Homozygous loss of autism-risk gene CNTNAP2 results in reduced local and long-range prefrontal functional connectivity. Cereb. Cortex 28, 1141-1153 (2018).

21. Pagani, M. et al. Deletion of autism risk gene Shank3 disrupts prefrontal connectivity. J. Neurosci. 39, 5299-5310 (2019).

22. Haberl, M. G. et al. Structural-functional connectivity deficits of neocortical circuits in the $\mathrm{Fmr}^{-1 /}$ mouse model of autism. Sci. Adv. 1, 1500775 (2015).

23. Zerbi, $\mathrm{V}$. et al. Dysfunctional autism risk genes cause circuit-specific connectivity deficits with distinct developmental trajectories. Cereb. Cortex 28, 2495-2506 (2018)

24. Sakagami, H. et al. IQ-ArfGEF/BRAG1 is a guanine nucleotide exchange factor for Arf6 that interacts with PSD-95 at postsynaptic density of excitatory synapses. Neurosci. Res. 60, 199-212 (2008).

25. Petersen, A., Brown, J. C. \& Gerges, N. Z. BRAG1/IQSEC2 as a regulator of small GTPase-dependent trafficking. Small GTPases 11, 1-7 (2018).

26. Murphy, J. A., Jensen, O. N. \& Walikonis, R. S. BRAG1, a Sec7 domain-containing protein, is a component of the postsynaptic density of excitatory synapses. Brain Res. 1120, 35-45 (2006).

27. Brown, J. C. et al. Bidirectional regulation of synaptic transmission by BRAG1/ IQSEC2 and its requirement in long-term depression. Nat. Commun. 7, 11080 (2016).

28. Rogers, E. J. et al. An IQSEC2 mutation associated with intellectual disability and autism results in decreased surface AMPA receptors. Front. Mol. Neurosci. 12, 43 (2019).

29. Shoubridge, C. et al. Mutations in the guanine nucleotide exchange factor gene IQSEC2 cause nonsyndromic intellectual disability. Nat. Genet. 42, 486-488 (2010).

30. Kalscheuer, V. M. et al. Novel missense mutation A789V in IQSEC2 underlies Xlinked intellectual disability in the MRX78 family. Front. Mol. Neurosci. 8, 85 (2016).

31. Mignot, C. et al. IQSEC2-related encephalopathy in males and females: a comparative study including 37 novel patients. Genet. Med. 21, 837-849 (2019).

32. Zipper, R. et al. Developmental progression of intellectual disability, autism, and epilepsy in a child with an IQSEC2 gene mutation. Clin. Case Rep. 5, 1639-1643 (2017).

33. Jabarin R. et al. Precision medicine for the rescue of specific impairments in social behavior associated with the A350V Iqsec2 mutation. bioRxiv https://doi. org/10.1101/2020.11.04.368282 (2020).

34. Guo, Z. V. et al. Procedures for behavioral experiments in head-fixed mice. PLOS ONE 9, 88678 (2014)

35. Moy, S. S. et al. Sociability and preference for social novelty in five inbred strains: an approach to assess autistic-like behavior in mice. Genes Brain Behav. 3, 287-302 (2004).

36. Lein, E. S. et al. Genome-wide atlas of gene expression in the adult mouse brain. Nature 445, 168-176 (2007).

37. Oh, S. W. et al. A mesoscale connectome of the mouse brain. Nature $\mathbf{5 0 8}$ 207-214 (2014).

38. Andersson, J., Jenkinson, M., \& Smith, S. Non-linear registration, aka Spatial normalisation. FMRIB Technial Report TR07JA2 (FMRIB Centre, Oxford, United Kingdom, 2007). 
39. Harris, J. A. et al. The organization of intracortical connections by layer and cell class in the mouse brain. bioRxiv https://doi.org/10.1101/292961 (2018).

40. Asleh, J. et al. Brain-wide structural and functional disruption in mice with oligodendrocyte-specific Nf1 deletion is rescued by inhibition of nitric oxide synthase. Proc. Natl Acad. Sci. USA 117, 22506-22513 (2020).

41. Bergmann, E., Gofman, X., Kavushansky, A. \& Kahn, I. Individual variability in functional connectivity architecture of the mouse brain. Commun. Biol. 3, 738 (2020).

42. Uludağ, K., Müller-Bierl, B. \& Uğurbil, K. An integrative model for neuronal activity-induced signal changes for gradient and spin echo functional imaging. Neuroimage 48, 150-165 (2009).

43. Yacoub, E., Harel, N. \& Uğurbil, K. High-field fMRI unveils orientation columns in humans. Proc. Natl Acad. Sci. USA 105, 10607-10612 (2008)

44. Power, J. D. et al. Methods to detect, characterize, and remove motion artifact in resting state fMRI. Neuroimage 84, 320-341 (2014).

45. Grandjean, J. et al. Common functional networks in the mouse brain revealed by multi-centre resting-state fMRI analysis. Neuroimage 205, 116278 (2020).

46. Grandjean, J. et al. Structural basis of large-scale functional connectivity in the mouse. J. Neurosci. 37, 8092-8101 (2017).

47. The jamovi project (2020).

48. Benjamini, Y. \& Yekutieli, D. The control of the false discovery rate in multiple testing under dependency. Ann. Stat. 29, 1165-1188 (2001).

49. Seo, S. A Review and Comparison of Methods for Detecting Outliers in Univariate Data Sets (2006)

50. Ellegood, J. et al. Clustering autism: using neuroanatomical differences in 26 mouse models to gain insight into the heterogeneity. Mol. Psychiatry 20, 118-125 (2015).

51. Ecker, C., Bookheimer, S. Y. \& Murphy, D. G. M. Neuroimaging in autism spectrum disorder: brain structure and function across the lifespan. Lancet Neurol. 14, 1121-1134 (2015)

52. Melozzi, F. et al. Individual structural features constrain the mouse functional connectome. Proc. Natl Acad. Sci. USA 116, 26961-26969 (2019).

53. Peça, J. \& Feng, G. Cellular and synaptic network defects in autism. Curr. Opin. Neurobiol. 22, 866-872 (2012).
54. Hinze, S. J. et al. Incorrect dosage of IQSEC2, a known intellectual disability and epilepsy gene, disrupts dendritic spine morphogenesis. Transl. Psychiatry 7, e1110 (2017).

55. Sah, M. et al. Altered excitatory transmission onto hippocampal interneurons in the IQSEC2 mouse model of X-linked neurodevelopmental disease. Neurobiol. Dis. 137, 104758 (2020).

56. Myers, K. R. et al. Arf6-GEF BRAG1 regulates JNK-mediated synaptic removal of glua1-containing AMPA receptors: a new mechanism for nonsyndromic $X$ linked mental disorder. J. Neurosci. 32, 11716-11726 (2012).

57. Piven, J., Elison, J. T. \& Zylka, M. J. Toward a conceptual framework for early brain and behavior development in Autism. Mol. Psychiatry 22, 1385-1394 (2017).

58. Jackson, M. R. et al. Heterozygous loss of function of IQSEC2/lqsec2 leads to increased activated Arf6 and severe neurocognitive seizure phenotype in females. Life Sci. Alliance 2, e201900386 (2019).

59. Di Martino, A. et al. Aberrant striatal functional connectivity in children with autism. Biol. Psychiatry 69, 847-856 (2011).

60. Balsters, J. H., Mantini, D. \& Wenderoth, N. Connectivity-based parcellation reveals distinct cortico-striatal connectivity fingerprints in Autism Spectrum Disorder. Neuroimage 170, 412-423 (2018).

61. Peça, J. et al. Shank3 mutant mice display autistic-like behaviours and striatal dysfunction. Nature 472, 437-442 (2011).

62. Wang, W. et al. Striatopallidal dysfunction underlies repetitive behavior in Shank3-deficient model of autism. J. Clin. Invest. 127, 1978-1990 (2017).

63. Delmonte, S. et al. Social and monetary reward processing in autism spectrum disorders. Mol. Autism 3, 7 (2012).

64. Guo, B. et al. Anterior cingulate cortex dysfunction underlies social deficits in Shank3 mutant mice. Nat. Neurosci. 22, 1223-1234 (2019).

65. Ullman, M. T. \& Pullman, M. Y. A compensatory role for declarative memory in neurodevelopmental disorders. Neurosci. Biobehav. Rev. 51, 205-222 (2015).

66. Basso, A., Gardelli, M., Grassi, M. P. \& Mariotti, M. The role of the right hemisphere in recovery from aphasia. Two case studies. Cortex 25, 555-566 (1989).

67. Livingston, L. A. \& Happé, F. Conceptualising compensation in neurodevelopmental disorders: Reflections from autism spectrum disorder. Neurosci. Biobehav. Rev. 80, 729-742 (2017). 